\title{
DOSAGE DU SEL MARIN DANS LE FROMAGE,
}

\author{
par B. van der BURG, \\ Professeur de laiterie, à Wageningen (Hollande).
}

Le sel joue un rôle très important dans le fromage. Il n'y a pas. seulement une influence directe sur la saveur du fromage, mais aussi indirecte par son action sélective sur les microorganismes se trouvant dans le fromage récemment préparé et ayant un degré de sensibilité différente pour le sel marin. Il s'en suit que le procès de maturation. est influencé par la concentration du sel dans le sérum du fromage. En outre, le sel influence la structure de la pâte du fromage. Car, à côté de l'acidité du sérum, la concentration du sel a une influence notable sur le gonflement de la paracaséine comme l'a démontré M. van Daм (1). Ce gonflement et ce qui s'en suit, la plasticité de la pâte, sont maximum en présence d'un sérum à environ $\check{5} \%$ de sel marin. En effet, M: van DAM a trouvé que le fromage avec une belle pâte plastique contient environ ${ }^{\circ} \%$ de sel dans le sérum.

Dans l'exumen de 93 fromages gras, tous à belle pâte plastique, nous avons trouvé une moyenne de $4,3 \check{\%} \%$ de sel dans le sérum $(1,87 \%$ dans la pâte), les extrêmes étant de $2,8 \%$ et $7,8 \% ; 70 \%$. des chiffres étant compris entre $3,8 \%$ et $5 \%$.

En présence du fait que le sel exerce une influence sur la saveur, sur le procès de maturation et sur les propriétés physiques du fromage, il est évident que dans une exploitation conduite rationnellement on sent le besoin d'avoir à sa disposition une méthode facile pour la détermination de la concentration du sel dans le fromage afin de pouvoir contrôler le salage.

Pour les fromages, tels que le fromage de Frise (épicé), le cheddar et le cheshire (nous nous bornons aux fromages fabriqués en Hollande) qui sont salés dans le caillé avant la mise dans. les moules, la quantité de sel à ajouter peut être pesée, mais les fromages de Gouda et d'Edam ne sont salés qu'après moulage et pressage, soit en les trempant pendant quelque temps dans une saumure, soit en les trottant de temps en temps avee du sel pendant quelques jours. Ce dernier procédé est encore d'usage assez courant pour la fabrication du fromage d'Edam dans les fermes et les petites fromageries.

Le pouvoir absorbant du fromage pour le sel est influencé par-

(1) Opstellen over moderne Zuivelchemie, $2^{e}$ édition (1922), p. 105 et suivantes; publié par le Algemeene Nederlandsche Zuiveebond, s' Gravenhage. 
plusieurs facteurs, par exemple, par le pourcentage de sérum, par le volume et la forme du fromage, par le pourcentage de graisse dans le caillé, par la température de la saumure ou du salage et par la concentration de la saumure. Une eonnaissance approfondie et surtout beaucoup de routine sont indispensables pour conduire cette opération de façon à obtenir la meilleure concentration de sel. Un contrôle chimique régulier serait d'un grand appui pour le fabricant de fromage.

Dans l'été 1920, un consortium de fabricants de fromages me demandait si je ne connaissais pas une méthode de dosage du sel dạns le fromage moins compliquée que le procédé fastidieux et conteux des cendres. Chose assez remarquable, dans aucun traité ou publication, je n'ai trouvé une méthode semblable à celle qui me fut demandée. Je leur ai recommandé le procédé suivant,qu , pour autant que je sache,n'a pas encore été publié.

On prend un échantillon du fromage et on le réduit en poudre dans un petit moulin. On mélange bien, on prend $4 \mathrm{gr}$. de fromage qu'on introduit dans un ballon gradué de $100 \mathrm{~cm}^{3}$, on y ajoute ŏ0 à $60 \mathrm{~cm}^{3}$ d'eau chaude et $10 \mathrm{~cm}^{3}$ de soude caustique (solution environ normale), on agite de temps en temps jusqu'à ce que le fromage soit complètement dissous. On laisse refroidir jusqu'à la température ambiante, puis on ajoute $10 \mathrm{~cm}^{3}$ d'acide nitrique $(6 \mathrm{~N}$. de p. s. \pm 1.2$)$ et l'on complète avec de l'eau distillée à $100 \mathrm{~cm}^{3}$. Après avoir agité on filtre en se servant d'un filtre sec, on prend $50 \mathrm{~cm}^{3}$ du liquide filtré et y ajoute $15 \mathrm{~cm}^{3}$ d'une solution normale décime de nitrate d'argent. L'excès d'argent est titré par une solution normale décime de sulfocyanure de potassium après addition de $1 \mathrm{~cm}^{3}$ d'une solution saturée d'alun de fer et sans élimination du chlorure d'argent formé.

En soustrayant du nombre de $\mathrm{cm}^{3}$ de la solution de nitrate d'argent celui du témoin et en multipliant le chiffre trouvé par 0,2920 , on obtient le pourcentage de sel présent dans le fromage. La correction pour le témoin ne peut pas être négligée, parce qu'il se peut que la soude caustique employé contienne du chlore.

Pour simplifier le calcul, on peut prendre $3 \mathrm{gr} ., 9$ au lieu de $4 \mathrm{gr}$. de fromage. Dans ce cas, on trouve le pourcentage de sel en multiliant le nombre de $\mathrm{cm}^{3}$ de la solution de nitrate d'argent par 0,3 .

Dans mon laboratoire, nous avons comparé cette méthode directe avec la méthode des cendres. Nous donnons les résultats de cette comparaison dans le tableau suivant, qui démontre que la méthode par titrage direct donne le pourcentage de sel avec une précision suffisante. 


\title{
Pourcentage de sel marin dans le fromage.
}

\begin{tabular}{|c|c|c|c|c|c|}
\hline $\mathrm{N}^{o s}$ & $\begin{array}{c}\text { Méthode } \\
\text { v. d.B. }\end{array}$ & $\begin{array}{l}\text { Méthode } \\
\text { des cendres }\end{array}$ & $\mathrm{N}^{\circ s}$ & $\begin{array}{l}\text { Méthode } \\
\text { v. d. B. }\end{array}$ & $\begin{array}{c}\text { Méthode } \\
\text { des cendres }\end{array}$ \\
\hline- & - & - & - & - & - \\
\hline \multirow{8}{*}{1} & 3,05 & 2,99 & 4 & 1,75 & 1,81 \\
\hline & 3,05 & & 5 & 2,05 & 2,12 \\
\hline & 3,10 & & 6 & 2,15 & 2,14 \\
\hline & 3,05 & & 7 & 1,70 & 1,78 \\
\hline & 3,05 & & 8 & 200 & 2,00 \\
\hline & 310 & & 9 & 1,85 & 1,96 \\
\hline & 2,95 & 295 & 10 & 1,75 & 1,75 \\
\hline & 2,95 & 3,01 & 11 & 1,60 & 1,69 \\
\hline \multirow[t]{6}{*}{2} & 2,95 & & 12 & 175 & 1,79 \\
\hline & 2,95 & & 13 & 1,40 & 1,39 \\
\hline & 2,95 & & 14 & 1,85 & 1,96 \\
\hline & 3,25 & 323 & 15 & 1,95 & 1,96 \\
\hline & 3,25 & 3,23 & 16 & 1,90 & 1.94 \\
\hline & 3,15 & 3,22 & 17 & 1,75 & 1,76 \\
\hline \multirow{4}{*}{3} & 315 & 3,21 & 18 & 1,90 & 1,92 \\
\hline & 3,15 & 3,21 & 19 & 1,85 & 1,84 \\
\hline & 3,15 & $\begin{array}{l}3,21 \\
3,23\end{array}$ & & & \\
\hline & $\begin{array}{l}3,15 \\
3,15\end{array}$ & $\begin{array}{l}0,20 \\
3,22\end{array}$ & & & \\
\hline
\end{tabular}

Laboratoire de laiterie de la Landbouwhoogeschool.

(Wageningen, mai 1923).

\section{GONSIDÉRATIONS SUR LA LÉGISLATION HYGIÉNIQUE DU LAIT,}

\author{
par H. KUFFERATH,
}

Ingénieur Agricole (Gembloux). - Docteur ès Sciences.

Directeur du Laboratoire intercommunal de l'Agglomération Bruxelloise.

Il est remarquable de constater que la législation laitière ait si peu profité jusqu'ici de la révolution d'idées que l'œuvre de Pasteur a accomplie. La médecine humaine et vétérinaire, la technologie laitière sont tout acquises au mouvement scientifique déterminé par l'étude des maladies et des fermentations. Il est à espérer que le Centenaire de Pasteur aura pour effet d'attirer sur les problèmes de l'hygiène l'attention des pouvoirs administratifs et législatifs.

Que le monde du Palais, que l'Administration n'acceptent ces idées 
nouvelles qu'avec lenteur, rien d'étonnant. La législation, toujours prudente ne s'engage dans des voies nouvelles qu'à bon escient, à cause des problèmes complexes que soulèvent des textes nouveaux et non éprouvés.

C'est ce qui explique psychologiquement le peu de hate de légiférer sur l'hygiène du lait. La loi ne sanctionne que des faits bien établis et c'est aux milieux familiarisés avec la pratique de l'hygiène à aller de l'avant, à faire des propositions. Les médecins, les vétérinaires, les Laboratoires d'analyse des denrées ont par conséquent à prendre l'initiative des mesures nouvelles.

Mais une législation telle que celle de l'hygiène ne peut s'établir en une fois. Avant d'être constituée, elle doit passer par une période transitoire qui marque l'étape entre l'ancienne législation et celle de l'avenir. Il est nécessaire, et même indispensable,que dans l'établissement de la législation hygiénique nouvelle, on tienne compte de l'ancienne législation qui existe de fait et de droit. C'est là une diffieulté du problème ; elle n'est pourtant pas insoluble.

Les législations variant de pays à pays, chaque cas doit être examiné à part et l'on ne peut indiquer que les lignes générales de la réforme à accomplir.

Nous exposerons le cas particulier de la législation belge et des efforts faits pour donner satisfaction aux besoins nouveaux de l'hygiène.

Notons de prime abord que les eommunes ont les droits les plus absolus en matière d'hygiène, ce n'est qu'en second lieu, que le gouvernement et les provinces ont des obligations en ce domaine. Nous ne connaissons pas de dispositions provinciales relatives aux questions d'hygiène des denrées alimentaires et du lait en particulier. Il ne reste donc en Belgique, que les lois et arrêtés du Gouvernement et les ordonnances communales,

Voyons d'abord, dans les lois et arrêtés généraux, les dispositions applicables au point de vue hygiénique. Ce sont d'une part des articles du Code pénal, d'autre part la loi du 4 août 1890 et l'arrêté du 10 novembre 1894. Déjà l'énoncé des textes des lois et arrêtés montre que les bases de la législation remontent à trente ans.

Les articles $\mathrm{n}^{0 \text { s }} 45 \% 4,455 \%, 456$ du Code pénal Belge sont libellés comme suit :

Art. 464. - Celui qui aura mêlé ou fait mêler, soit à des comestibles, ou des boissons, soit à des substances ou denrées alimentaires quelconques, destinés à être vendus ou débités, des matières qui sont de nature à donner la mort ou à altérer gravement la santé, sera puni d'un emprisonnement de six mois à cinq ans et d'une amende de deux cents franes à deux mille francs. 
Art. 455 - Sera puni des peines portées à l'article précédent :

Celui qui vendra, débitera ou exposera en vente des comestibles, boissons, substances ou denrées alimentaires quelconques, sachant qu'ils contiennent des matières de nature à donner la mort ou à altérer gravement la santé ;

Celui qui aura vendu ou procuré ces malières, sachant qu'elles devaient servir à falsifier des substances ou denrées alimentaires.

Art. 456. - Sera puni d'un emprisonnement de trois mois à trois ans, et d'une amende de cent franes à mille franes, celui qui aura dans son magasin, sa boutique ou en tout autre lieu, des comestibles, boissons, denrées ou substances alimentaires, destinées à ètre vendus ou débités, sachant qu'ils contiennent des matières de na ure à donner la mort ou à altérer gravement la santé.

Comme on le voit, ces textes sont très généraux et ne sont applicables que dans des cas spéciaux où les comestibles ou boissons (le lait par conséquent également) renfermeraient des matières de nature à donner la mort ou à altérer gravement la santé. Il n'est pas douteux que du lait contenant des microbes pathogènes et ayant causé mort d'homme ou maladie grave tomberait sous l'application de ces articles. Il serait même possible de l'appliquer pour les cas de maladie de carence.

Pourtant, les cas d'application de ces articles du Code sont exceptionnels. Dans la lutte contre les maladies transmissibles par ingestion de lait, on peut pourtant en faire usage. Voici dans quelles conditions : ces articles, notamment l'article 4อ̆อ̆, exigent que l'auteur du délit ait connaissance du danger qu'il cause, " sachant qu'ils contiennent des matières de nature à donner la mort ou à altérer gravement la santé "). Si un fermier vend du lait de vache malade (mammite aiguë, par exemple), après avoir été prévenu par un service d'analyse ou d'inspection que ce lait renferme des microbes pathogènes et qu'un accident se produit chez un consommateur, il est certain que l'article 4 อั est applicable.

En pratique, cet article du Code pénal peut être très utile pour couper court à des faits délictueux et constitue une menace très grave pour ceux qui croirait pouvoir le mépriser.

L'article 5 de la loi du 4 aont 1890 relative à la falsification des denrées alimentaires est libellé comme suit :

Article 5 - Le paragraphe $2^{\circ}$ et les alinéas 1 et 2 de l'article 561 du Code pénal sont modifiés dans les termes suivants :

20 Ceux qui auront vendu, débité ou exposé en vente des comestibles, boissons, denrées ou substances alimèntaires quelconques gâtés, corrompus ou déclarés nuisibles par un règlement de l'administration générale, provinciale ou communale : 
$3^{\circ}$ Ceux qui, sans l'intention frauduleuse exigée par l'article 500, auront vendu, débité ou exposé en vente des comestibles, boissons, denrées ou substances alimentaires quelconques falsifiés ou contrefaits.

Les comestibles, boissons, denrées ou substances alimentaires gâtés, corrompus, nuisibles, falsifiés au contrefaits qui seront trouvés en la possession du coupable seront saisis et confisqués.

Cet article est d'application plus fréquente, il suffit que les aliments soient gâtés, corrompus ou déclarés nuisibles par un règlement de l'Administration générale, provinciale ou communale. Au point de vue hygiénique, il est possible de déterminer, par examen bactériologique et microscopique spécialement, l'état gâté ou corrompu des aliments ; de plus, il suffit qu'un règlement communal déclare nuisible un état bien défini du lait, par exemple : présence de germes microbiens, présence de souillures dépassant un certain taux, présence de sang, pour que l'art. こّ de la loi du 4 aoùt soit applicable à ce lait.

On le voit, la portée de cet artiele peut être très grande et il accorde aux pouvoirs généraux, provinciaux et communaux, le droit de déterminer le caractère nuisible d'un produit alimentaire. Sur ce point, l'initiative des communes peut se manifester dansl'intérêt de la santé publique et introduire dans la législation des principes nouveaux conformes aux préceptes de l'hygiène.

L'arrêté royal du 10 décembre 1890 (complété par celui du 15 septembre 1891) vise l'emploi des substances métalliques mises en contact avec les aliments.

Au point de vue de l'hygiène, cet arrêté peut être appliqué à l'examen des étamages des cruches, aux conduites et récipients divers utilisés dans les laiteries.

L'arrêté royal du 18 novembre 1894 est certainement l'arrêté belge le plus intéressant au point de vue de l'hygiène. Son article 2 mérite d'être reproduit :

" Il est interdit d'une manière absolue de vendre, d'exposer en vente, de détenir ou de transporter pour la vente, pour l'alimentation humaine, sous quelque dénomination que ce soit:

10. Du lait additionné d'eau ou de substances étrangères quelconques, telles quagents de conservation ;

$2^{\circ} \mathrm{Du}$ lait colostral; du lait altéré par des microgermes ou des produits infectieux (lait acide, visqueux, putride, amer, bleu, rouge, etc.), soit à raison d'un état anormal, ou d'une alimentation défectueuse du bétail, soit par suite d'une tenue défectueuse de l'étable, de la laiterie ou des ustensiles de transport, soit par toute autre cause, telles que des manipulations effectuées pardes personnes peu soigneuses ;

$3^{\circ} \mathrm{Du}$ lait provenant d'animaux aux aliments desquels auraient été mêlées. 
des plantes vénéneuses, d'animaux médicamentés à l'aide de substances toxiques ou d'animaux atteints de maladies contagieuses ou infectieuses, telles que tuberculose (pommelière), rage, fièvre aphteuse (cocotte), fièvre charbonneuse, charbon symptoratique, pyohémie, septicémie, diphtérie, mammites aiguës et mammite chronique avee suppuration, jaunisse, etc...

(Arr. roy.du 31 octobre 1898, Mon. du 27 nov.). Toutefois, le lait des vaches atteintes de fièvre aphteuse pourra être livré au commerce après avoir été soumis à l'action de la chaleur d'après un procédé agréé par notre Ministre de l'Agriculture et des Travaux publics

Une circulaire interprétative de 23 novembre 1894 signale que, si certains pays interdisent la vente du lait provenant de bêtes malades, il n'y a lieu de considérer que le lait au point de vue des germes ou principes nuisibles, ptomaïnes, pus, etc... qu'il peut contenir. L'analyse bactériologique pourra, dans certains eas, venir en aide à l'inspection ordinaire, pour le diagnostic des maladies.

La circulaire précise ; « Des règlements étrangers interdisent la vente du lait provenant de maisons où règnent des maladies contagieuses telles que la fièvre typhoïde, la variole, la scarlatine, la diphtérie. Mais le Gouvernement voulant éviter un excès de rigueur, croit pouvoir se borner à recommander instamment l'isolement du lait d'avec le malade, les personnes qui le soignent, les locaux.qu'il occupe, ainsi que les matières ou objets s'étant trouvés en contact avec lui. »)

" De même, si des animaux de la ferme sont atteints des affections indiquées au $3^{\circ}$ de l'article 2 , les personnes soignant ces animaux devront s'abstenir de manipuler le lait et on évitera de le laisser séjourner dans des locaux non convenablement isolés des lieux occupés par les dits animaux ou par des matières ou objets en contact avec eux. )

( Il est également recommandé de ne pas laver les récipients à lait avee de l'eau qui ne serait pas à l'abri de toute contamination par les germes des maladies sus-visées. ”

Il n'y a qu'une chose à regretter, e'est que les recommandations insérées dans la circulaire interprétative n'aient pas été données sous forme légale, ce ne sont qué des recommandations, et en réalité, toutes judicieuses qu'elles soient, elles sont sans effet pratique.

En réalité, le paragraphe de l'alinéa $2^{\circ}$ de l'Arrêté royal du 25 novembre 1894 n'a pas reçu d'application depuis sa publication jusqu'il y a un an environ, pour la raison que l'on n'avait pas de méthode pratique de décéler les souillures du lait, indices d'une tenue défectueuse de l'étable, de la malpropreté des manipulations, etc... Cette lacune, qui a rendu l'Arrêté inopérant, fut comblée par l'emploi des filtres Gerber qui permettent d'apprécier la saleté du 
lait. Grâce aux dispositifs imaginés par le Laboratoire Intercommunal de Bruxelles (mise des échantillons de filtration sous enveloppe à lucarne, du modèle que nous avons exposé à l'exposition Pasteur de Strasbourg) la justice a eu à sa disposition une preuve de souillure du lait et a pu appliquer l'Arrêté. Les sanctions prises en suite des dispositions légales ont facilité, d'une façon inespérée, la campagne faite par le Laboratoire Intercommunal pour obtenir que l'agglomération bruxelloise reçoive un lait propre. Il nous suffira de dire qu'en moins d'un an, le Laboratoire Intercommunal de Bruxelles est parvenu à abaisser le taux pour cent de laits sales de $90 \%$ (janvier 1922) à $29 \%$ (décembre 1922). Actuellement, la quantité de laits sales débités aux consommateurs bruxellois est presque nulle.

Ajoutons que, simultanément à cette grande amélioration de la propreté du lait, la proportion des laits falsifiés est tombée, grâce à la surveillance continuelle du lait faite à Bruxelles, à environ 2 ou $3 \%$ et encore les falsifications sont-elles en général minimes : mouillages inférieurs à $10 \%$, écrémages légers. Si l'on compare les résultats obtenus au bout de quelques années, à la situation dans d'autres agglomérations belges ou dans d'autres pays, on se rendra compte de l'eflicacité des mesures prises. Pour ne prendre qu'un exemple récent (1) en Lorraine, M. SA вAті́́ a constaté $43 \%$ de laits falsifiés en 1920 et $23 \%$ en 1922, les mouillages étant les principales fraudes constatées.

Nous sommes persuadés, par l'expérience que nous vivons journellement, que le système de contrôle du lait pratiqué par le Laboratoire Intercommunal est applicable à toute agglomération aussi importante qu'elle soit, avec les mêmes résultats que nous enregistrons à Bruxelles.

Si pourtant la législation gouvernementale belge est peu développée et présente des lacunes, il est néanmoins prouvé qu'elle peut donner des résultats appréciables.

Nous avons vu que, suivant la législation belge. les Provinces et Communes ont le droit de prendre des Arrêtés. La législation provinciale est inexistante, mais quelques communes ont pris des Arrêtés concernant le commerce du lait. C'est ainsi que Saint-Gilles-lezBruxelles, a pris dès 1912 un règlement inspiré par les délibérations de la Commission Permanente du lait.

Ce règlement a été adopté dans les termes suivants :

Le Conseil Communal,

Considérant qu'il importe, dans l'intérèt de la santé publique, de prendre des mesures en vue d'empècher la vente de lait capable d'engendrer des maladies chez le consommateur;

(1) Annales des Falsifications, 1923, n 175, page 242. 
Vu le rapport présenté sur cette affaire et le résultat du vote unanimement favorable tant aux conclusions du dit rapport, qu'aux amendements proposés par MM. les Conseillers Delange et Van Driessche;

Vu la loi du 4 août 1890 sur la folsification des denrées alimentaires ;

Vu l'Arrêté royal du 18 novembre 1894 relatif à l'application du règlement sur le commerce du lait;

Vu l'article 78 de la loi communale;

\section{Ordonne:}

Article premier. - Il est interdit de transporter, de colporter, d'exposer en vente, de détenir pour la vente, sur le territoire de la commune de SaintGilles, du lait provenant d'animaux atteints d'affections dont les germes ou leurs toxines rendent le lait nocif, du lait malpropre, mal conservé. Les producteurs, les colporteurs, les vendeurs, les débitants ont à établir la preuve par certificat émanant d'un vétérinaire du lieu où est étahlie la vacherie ou la laiterie, que le lait provient de vaches saines et bien entretenues.

Ce certificat, datant au plus de six mois, doit être produit à toute réquisition des agents de l'autorité.

Art. 2. - Le nom et l'adresse du laitier sont inscrits en caractères bien lisibles sur le véhicule ou sur les récipients servant au transport du lait destiné à la vente.

Art. 3.- Le transport simultané, dans les mêmes véhicules, de substances ou objets quelconques pouvant souiller ou altérer le lait ou qui pourraient, éventuellement, lui être mélangés, est strictement interdit. Les déchets culinaires, les légumes, fruits, etc., sont tolérés à condition d'être isolés des récipients qui contiennent le lait. A cet effet, ces déchets sont placés dans des caisses mobiles suspendues sous les véhicules.

Les récipients doivent être hermétiquement fermés pendant le transport et tenus parfaitement propres tant à l'extérieur qu'à l'intérieur.

Art. 4. - Les infractions aux dispositions de la présente ordonnance sont punies d'une amende de 1 à 25 francs et d'un emprisonnement de 1 à 7 jours, séparément ou cumultativement, selon les circonstances.

Arrêté par le Conseil communal en séance du 10 octobre 1912.

Pris pour notification par la députation permanente, le 23 óctobre 1912.

Publié le 28 novembre 1912.

Malheureusement, l'application de ce règlement s'est butée à des difficultés insurmontables. L'obligation de faire accompagner le lait des certificats de bonne santé des laiteries productrices est quasi impossible.

Cette idée, très belle en théorie,est impraticable dans le commerce.

S'imagine-t-on une firme, livrant 10.000 litres de lait journellement, devant foumir régulièrement les certificats de 1.000 à 1.100 vaches laitières à chacun de ses camionneurs, revendeurs et débitants au détail, de manière qu'à toutes réquisitions, ces certificats puissent être produits aux inspecteurs. Quelle est la valeur qu'une administration peut accorder à de tels certificats?Il y a des vétérinaires qui 
se sont refusé d'engager leur responsabilité professionnelle, car, une vache peut être aujourd'hui bien portante et présenter quelques jours après une infection aiguë, et il se pourrait très bien que, sous le couvert d'un certificat en bonne et due forme, un marchand se croit à l'abri de tout reproche. Nous avons d'ailleurs eu un cas semblable: une Iaiterie voulant se mettre en règle avec le règlement de Saint-Gilles, avait demandé le certificat à son vétérinaire lequel avait déclaré que tout était pour le mieux dans sa région et qu'à sa connaissance la fièvre aphteuse ne régnait pas dans la contrée.Administrativement, la laiterie était eouverte par son certificat; en réalité, deux jours après le dépôt de cette pièce, les services d'analyse du Laboratoire intercommunal découvrent un lait à streptocoques; le hasard veut préeisément que ce lait provienne de la laiterie en question.

Des certificats vétérinaires ne pourraient avoir de valeur que si le gouvernement décrétait par une loi l'inspection obligatoire périodique des vaches laitières et créait un corps d'inspecteurs vétérinaires.

D'après des ealculs extraits d'un rapport sur la question, voté par le Syndicat vétérinaire liégeois, M. Jules Grandry, rapporteur, il faudrait pour la province de Liége, 13 inspecteurs; ce qui ferait pour la Belgique entière, environ 120 inspecteurs des vacheries et ils devraient visiter en moyenne 90 vaches par jour, en veillant à la santé des vaches, l'habitat, l'alimentation, la qualité de l'eau, le conservation et le transport du lait, la santé du personnel, etc., tous points qui seraient examinés par l'inspecteur. Evidemment le programme esquissé par les vétérinaires liégeois est séduisant, mais que donnerait-il en pratique? Pour chaque bête examinée, le certificat délivré par le vétérinaire servirait de laisser-passer et devrait être produit à toute réquisition. Ce système engendrerait une paperasserie formidable. L'agencement d'un tel service serait hors de proportion avec le résultat à obtenir.

Ce système serait aussi peu logique que si l'administration exigeait tous les quinze jours un certificat de bonne santé des habitants d'une région. II faudrait pour cela un personnel médical innombrable, un régime de contrôle dont les résultaț seraient vains et susciteraient des dépenses invraisemblables.

Le procédé de contrôler les bêtes à l'étable est rempli de diffieultés. Ce qui importe pour le eonsommateur ce n'est pas de savoir que la vache qui produit le lait qu'il boit est saine, c'est de savoir si ce lait est sans danger. Il importe donc que le contrôle du lait dans les lieux de consommation soit assez vigilant et bien agencé pour dépister tout lait, même suspect et l'interdire à la consommation. Quitte,lorsqu'un lait suspect a été repéré, à faire diligence pour retrouver son 
origine et faire alors examiner la vache productrice par le vétérinaire et exiger un certificat motivé et en règle.

C'est ce système que nous avons adopté au Laboratoire intercommunal. Son application ne présente aucune difficulté et, bien mieux, a fonctionné, depuis plusieurs mois, avec plein succès. Actuellement, toutes les grandes firmes de l'agglomération se sont soumises à ce système, le contrôle ainsi effectué est absolument gratuit et n'a causé aucun supplément de dépenses au budget du Laboratoire. Par ce procédé, nous sommes arrivés à contrôler la grande majorité des producteurs de lait alimentant Bruxelles, agglomération comptant plus de 800.000 habitants.

Il est évident que l'on se trouve encore, en Belgique, dans une période de transition entre l'ancienne législation répressive, assez complète pour les fraudes intéressant la chimie du lait et entre la législation bygiénique de l'avenir qui devrait s'efforcer d'ètre plus préventive que répressive. Nous savons bien qu'une formule concrête n'est pas facile à trouver, mais nous ne doutons pas que l'opinion publique, éclairée par les hommes compétents en la matière, ne finisse par avoir satisfaction.

Les exemples que nous avons produits montrent qu'un passage progressif de l'ancienne législation à la nouvelle, peut se faire sans heurts et que,tous comptes faits, nos codes ont des textes assez larges pour donner un appui à des idées scientifiques nouvelles.

4 Après avoir examiné le cas de l'application des idées d'hygiène à la réglementation belge du lait, nous allons reprendre le problème à un point de vue plus général. Nous n'avons ici que la prétention d'exposer quelques idées sur la matière, notre but étant bien plus de provoquer des discussions et des objections que de résoudre la question.

Demandons-nous le but que doivent viser les lois L'ancienne conception du Code Napoléon était d'assurer le libre développement des droits de l'homme et une répression complète des délits. Toute notre organisation judiciaire et administrative découle de cette conception. Mais les tendances nouvelles se sont fait jour; la relativité de la responsabilité humaine, les acquisitions de la psychologie, l'idée de la défense sociale opposée à celle de l'intérêt individuel, tout un ensemble de lois qui tendent à battre en brèche les principes établis par la grande Révolution française, sont des indices d'une mentalité nouvelle. D'après ces idées, la répression n'est plus l'unique moyen d'amélioration sociale, c'est même un moyen détestable et une source d'iniquité continuelle. La lutte contre l'ignorance, l'aide accordée par les Pouvoirs publics pour l'amélioration des conditions générales, la limitation de la liberté individuelle, l'importance des mesures d'hy- 
giène au point de vue social (création de Ministères de l'Hygiène), la prépondérance des facteurs économiques démontrée par le bouleversement actuel du monde, dépassent les cadres tracés par le Code qui nous régit et tendent vers de nouvelles mesures plus en rapport avec le développement de la science.

Il y a lieu de tenir compte d'une législation nouvelle. C'est ainsi qu'il n'est plus possible de considérer la législation laitière au point de vue exclusivement répressif. Si la recherche des délits continuera à être la raison des organismes de contrôle des denrées, leur mission semblerait plus belle s'ils n'avaient qu'à vérifier la bonne qualité des aliments. Actuellement, l'ambition de beaucoup d'inspecteurs est de pouvoir faire état d'un plus grand nombre de condamnations. C'est, si l'on voudra, une déformation protessionnelle qui pent conduire aux interventions les plus étranges. Tous les jours, des plaintes se font entendre sur la rigueur des tribunaux et l'on cite volontiers tels cas de condamnation injuste; on s'étonne que tel petit laitier soi frappé d'une amende écrasante pour lui, alors que tel autre, pour le même fait, s'en tire sans dommages réels. Et l'on pourrait multiplier les exemples.Pour éviter les critiques, pour faire régner une justice plus équitable, n'y a·t-il que la répression inexorable? Nous pensons que non.

Il faudrait instaurer un contrôle continuel, des vérifications multiples, et pour le lait rien n'est plis facile, de manière que les fraudeurs soient constamment sur le qui-vive. Il faul aussi que les inspecteurs aient à leur disposition des moyens sommaires de vérifications adéquats, qu'ils soient à même de ne laisser circuler que la marchandise de bon aloi et que tout lait suspect soit l'objet d'une enquête et de poursuites éventuelles. Le service d'inspection a done à jouer un rôle primordial, il doit constituer une sorte de crible ne retenant que les faits délictueux ou contraire à l'hygiène publique. En dépistant les fraudeurs, en les traquant sans rémission, en n'inquiétant pas les commerçants honnêtes, on atteindra des résultats autrement grands que par une répression aveugle.

Il y aurait lieu aussi d'apprendre aux intéressés à se mettre en garde contra des fournisseurs malintentionnés ou même négligents. Il y a lieu de faire l'éducation de beaucoup de laitiers qui connaissent. imparfaitement leur métier. C'est dans cet ordre d'idées que les services de prélèvement du Laboratuire Intercommunal apprennent à quiconque le demande, les procédés employés par les inspecteurs. pour dépister les falsifications. L'article 561, 3e du Code Pénal Belgeest une menace continuelle pour les détaillants qui négligent de vérifier le lait qu'ils reçoivent. Ils peuvent être condamnés pour avoir vendu une marchandise falsifiée ou contrefaite, tout en ignorant l'état. 
défectueux de cette marchandise. On voit le danger d'une telle dispotion. C'est pour cela que la vérification des denrées à la réception doit être faite par le réceptionnaire.

Dans le mème ordre d'idées nous avons préconisé auprès des firmes laitières l'installation de contrôle des fournitures. Les plus grandes difficultés ont été rencontrées pour faire accepter ces vues; non pas qu'elles n'étaient pas appréciées, mais par souci de concurrence,car les firmes qui se montraient trop exigeantes voyaient les producteurs les abandonner. Il y eut bien quelques difficultés an début, mais deux firmes inaugurèrent le système, leurs directeurs eurent le réel courage de tenir bon et de ne pas désespérer devant les insuccès de la première heure. On peut les en féliciter. Actuellement la plupart des grandes firmes laitières de Bruxelles vérifient la propreté du lait à l'arrivée et sont parvenues à recevoir un lait pour ainsi dire impeccable au point de vue des souillures. Ces résultats, qui font l'orgueil des firmes et qu'elles se plaisent à montrer à ceux qui les visitent, montrent l'importance que peuvent avoir des mesures de contrôle préventif.

Actuellement, les laits de mélange reçus par les laitiers sont envoyés au contrôle du Laboratoire Intercommunal régulièrement toutes les semaines. Un examen de ces laits (densité, graisse, épreuve de filtration, taux leucocytaire et examen microseopique, etc.) est fait et un rapport est envoyé avec remarques. Les laitiers qui se soumettent à ces vérifications donnent le nom des fournisseurs récolteurs. S'il y a, par exemple, une souillure trop forte du lait, une note est envoyée immédiatement au récolteur qui est mis en demeure de s'exécuter. Des contrôles à l'arrivée permettent de poursuivre l'enquête. Il en est de même dans le cas de taux leucocytaire anormal ou présence de streptocoques; on remonte à l'origine et grâce à une organisation minutieuse on parvient à dépister, en deux ou trois jours au maximum, les vaches suspectes. Il arrive que les récolteurs demandent à leur tour une vérification du lait qui leur est livré par les producteurs, de la sorte le contrôle du lait se fait automatiquement dès l'origine.

Finalement le producteur est contrôlé à son tour, et une vérifieation du lait au moment de la traite vient compléter le contrôle. Au besoin, les inspecteurs font une démonstration par filtration et engagent les fermiers à se conformer aux exigences du contròle.

Les résultats obtenus par ces divers moyens, qui viennent compléter la répression éventuelle des délits, sont des plus encourageants. C'est au point que, dans l'agglomération bruxelloise, les inspecteurs communaux et ceux du Laboratoire Intercommunal ne rencontrent presque plus de falsifications. 
D'ailleurs, nous avons trouvé un appui auprès de quelques bourgmestres de Communes rurales qui ont jugé utile d'avertir par voie d'affiches leurs concitoyens des conditions de salubrité du lait exigées par les services du contrôle laitier du Laboratoire Intercommunal. Divers directeurs de laiteries ont fait les mèmes recommandations aux producteurs, en leur distribuant des imprimés. Il est donc possible, même avec une législation incomplète, d'obtenir un ehangement dans la qualité et la valeur hygiénique des laits. On ne peut donc juger la valeur d'une législation d'après les textes. Et il est même à souhaiter que la rédaction des articles de la loi soit très générale et qu'ils puissent être appliqués à de nombreux cas. Nous convenons volontiers qu'il n'est pas commode de libeller des textes pareils, c'est là l'affaire de juristes rompus au métier.

On peut se demander si un texte général n'est pas préférable à une législation fouillée et précise, comme celle qui vient d'être publiée en Angleterre sur les laits Grade A, Grade A certified, Pasteurised Milk. Ces règlements fixent des normes bactériologiques, organisent l'inspection du bétail, décrètent la tuberculination, etc... Personnellement, nous applaudissons à ces mesures, mais que donnera en pratique ce système parfait en théorie ? Il est possible qu'avee l'esprit réaliste anglais, on obtienne là-bas, tout comme aux Etats-Unis où existe une législation analogue depuis plusieurs années, de bons résultats. Mais en Belgique, où l'équipement bactériologique des laboratoires de répression des fraudes, sauf le Laboratoire Intercommunal, est plutôt sommaire, une telle législation serait inefficace.

Le problème du contrôle hygiénique du lait devrait être envisagé à notre avis de la façon suivante : récolter le lait proprement de vaches saines et normales ; le lait aussitôt récolté devrait être refroidi et conservé proprement en dessous de $10^{\circ} \mathrm{G}$. Cette formule est peutêtre trop brève, trop peu détaillée, mais elle renferme l'essentiel de ce que l'on pourrait exiger dans l'intérêt du consommateur.

Il y a un point auquel on ne prête généralement que trop peu d'attention, e'est que, dans l'établissement d'une législation nouvelle, on doit tenir compte des conditions de la mentalité des classes agricoles et aussi des conditions économiques qui régissent le commerce laitier. Une législation qui méconnaîtrait ces éléments risquerait d'être sans effet.

Ajoutons, pour finir, une dernière remarque au sujet de l'organisation des services de Contrôle. Il y a le plus grand intérêt pour obtenir un résultat à ce que les services de prélèvement et d'analyse soient intimement unis dans un même organisme. Si une telle organisation est difficile pour un service gouvernemental, elle est facile à réaliser pour l'inspection communale ou régionale. Nous savons que des 
considérations de principes plaident contre cette union, mais les avantages à en tirer nous paraissent supérieurs, infiniment, aux raisons qui veulent que le chimiste travaille sur un simple numéro d'analyse.

\section{HYGIENE ET PRODUGTION DU LAIT SUR LES HAUTS-PLATEAUX DE L'ALGERIE,}

par

M. JEAN GANAC,

Vétérinaire-Sanitaire à Sétif. et

Considérations générales: - La production du lait a pris en Algérie, dans ces dernières années, une grande extension: lesstatistiques nous fournissent un chiffre de 1.093 .000 bâtes bovines ainsi réparties :

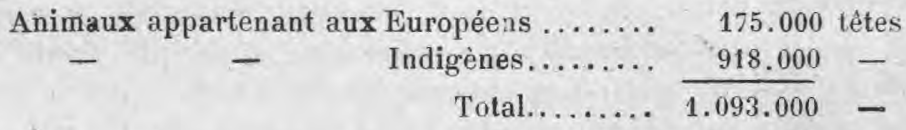

On ne connaît pas le nombre exact des vaches laitières ; le recensement en est assez difficile, toutefois, on peut dire que le plus grand nombre est exploité par les Européens.

Les races les plus diverses se trouvent en présence : celles de la Métropole qui ont été introduites, se sont pour la plupart acclimatées et donnent une moyenne de 2.600 litres de lait par au.

Parmi celles qui ont donné les meilleurs résultats, il faut citer les races de Schwytz, Tarentaise et Montbéliarde. Nous signalerons la rusticité de la race Tarentaise, qui, entretenue à peu près dans les mêmes conditions que la bête indigène, donne de bons rendements. La race Schwytz est plus exigeante, plus délicate ; la race Fribourgeoise donne des produits remarquables au point de vue laitier.

Les races indigènes, soit par sélection ou croisement avec les races précitées, arrivent à donner 1.800 à 2.000 litres de lait. La race de Guelma, sobre, rustique, dont l'élevage s'est étendu principalement dans la région des Hauts-Plateaux, réalise le type parfait pour l'Algérie ; sans aucun doute, par une sélection attentive, aidée de quelques croisements avec les races françaises, on arriverait à des résultats satisfaisants.

Dans les procés-verbaux de la Commission de l'Elevage,convoquée en 1914 par" le Gouvernement Génèral, nous trouvons.pour le choix 